covered with dry lint, and the bandage was re-applied. On Jane 2nd union by first intention was complete. The child was last seen at the beginning of July. The cicatrix then appeared to be very firm and the flannelette binder was

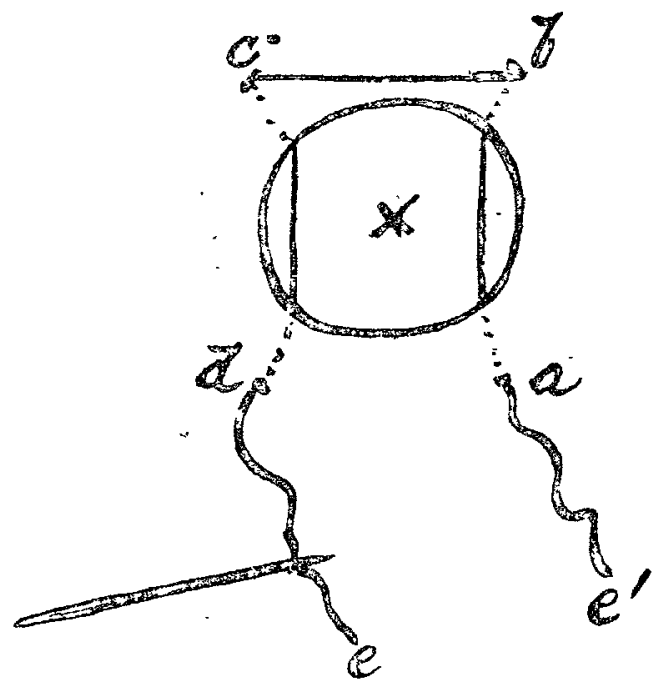

The freshened edge of the ulcer is represented by the circle. $x$, Floor of the ulcer lined by peritoneum. $a, b, c d$ indicate the course of the needle carrying the suture. $e, e^{\prime}$, Free ends of suture ready for tying.

discontinued. The infant's general condition was good and there was no trace of any bulging at the cicatrix.

Remarks by Mr. HERBERT.-Little is said in the textbooks both on surgery and on children's diseases about inflammation at the umbilicus and an unfavorrable prognosis is given. The inflammation runs much the same course as elsewhere and is doubtless caused by septic organisms. In the above case the condition was no doubt a septic one, the resulting ulceration being dependent upon its severity. It would be interesting to have had more particulars about the "core" which the mother said had come away previously to the ulceration manifesting itself. Three features in the case, however, seem to be noteworthy. 1 . The absence of any bulging at the site of the ulcer. This was doubtless due to the fibrous ring of the umbilicus being left more or less intact and capable of offering sufficient resistance to internal pressure. 2. The complete peeling off of the structures superficial to the peritoneum, leaving this membrane to form the floor of the resulting cavity. 3. The absence of toxic symptoms during the free employment of both carbolic and boric solutions, both of which have given rise to unpleasant symptoms in their use in infants and young children. I am indebted to Dr. Pinkerton for permission to publish this case.

\section{LUCEA HOSPITAL, JAMAICA.}

A CASE OF PROLAPSE OF THE RECTUM AND A CASE OF FIBROIDS OF THE UTERUS.

(Under the care of Dr. E. R. C. EARLE.)

THE case of prolapse of the rectum and sigmoid flexure described below may be looked upon as really an example of intussusception, for the upper part of the rectum had become invaginated into the lower. Many cases of prolapse commence in this manner, but so long as there is a sulcus between the protruding mass and the anus the case is one of intassusception. A case in many respects resembling the one appearing below was recorded by Dr. C. B. Kelsey, ${ }^{1}$ though the intussusception had existed for a longer time. The second of Dr. Earle's cases illustrates well the fact that of all forms of uterine fibroid the most easily removed is the pedunculated subperitoneal variety, and with modern asepsis the risk to life is but slight. In this case the size of the tumours was very great and "would have justified even more severe measures.

CASE 1. Prolapse of the rectum with inragination of the sigmoid flexure; recovery.-A feeble old coloured man, 89 years of age, was admitted into Lucea Hospital at 11.30 A.Mr. on Oct. 22nd, 1899, with a large tumour protruding from the anus. He stated that at 2 P.M. on the previous day, whilst straining at stool, he felt the bowel come down and that it bad remained in that condition ever since. On examination

1 The Post-Graduate, New York, August, 1895. it was seen that a large tumour, eight inches in length and between two and a half and three inches in diameter, protraded from the anus. The tumour consisted of prolapsed rectum and sigmoid flexure. It was conical in shape and slightly smaller at the lower end than at the middle; at the lower end there was an opening similar to a large os uteri, up which the finger could be passed. The surface of the tumour consisted of mucous membrane with concentric sulci ; it was dark red in colour and much congested and swollen, with small, dark, submucous hæmorrbages. It was not strangulated at the anus and there was no odour of gangrene. The anus was lax and the finger could with ease be passed up for two or tbree inches between the sphincter and prolapsed bowel all round the tumour. The patient complained of serere pain in the back but not in the tumour; he was very old, poorly nourished, and very weak.

At 12 noon he was put under chloroform and the tumour, which was thoroughly cleaned and warmed, was replaced gradually and slowly without any great difficulty, owing to the lax condition of the anus. There was a slight laceration in the posterior wall of the anus. Powdered tannic acia and a morphia suppository were put in the rectum and a perineal pad and band were applied. Hot-water bottles were placed at either side and the hips were raised on a pillow.

At 6 P.M. his condition was fairly good. He had slept a little and was in no great pain. Five minims of liquor opii sedativus were given after the operation and one and a half ounces of whisky in water. He passed a good night. On Oct. 23rd, early in the morning, a stool was passed containing serous bloody fluid and small blood.clots. The bowel was washed out with boric acid lotion and a five-grain suppository of tannic acid was inserted. The patient was comfortable and was stronger. At 5 P.M., after an enema of boric acid lotion, no fæcal matter came away; the fluid was dark-coloured and was mixed with shreds of mucous membrane. The tannic acid suppository was repeated. The patient rested well during the night. There was no action of the bowels; the pain in the back was relieved, but he had pain in the left shoulder and front of the chest. At 5 P.M. an enema was administered; the stool was very offensive. The suppository was given as before. On the 25 th he had slept well; the former pain was relieved, but be now had pain in the left hip. No stool had been passed during the night. The enema and suppository were administered morning and evening as before. On the 26th he had slept well. The bowels had acted once during the night; the stool was offensive. The treatment was continued. On the 27 th the patient passed three liquid stools, on the $28 \mathrm{th}$ and 29th two, and on the 30th and 31st one each day. There were no signs of prolapse and there was no pain. On the 31st he became very weak and low and had much difficulty in speaking, but he became stronger on Nov. 1st. He was discharged from the hospital on the 5 th.

CASE 2. Large fibroids of the uterus; removal; recovery.A coloured woman, aged 40 years, was admitted into Lucea Hospital on June 1st, 1899, complaining of great distension of the abdomen which had gradually increased during the previous three years. On examination the abdomen was found to be much distended. There was a large moveable tumour felt in the front and right side of the abdomen, passing into the right lumbar region and upwards under the liver, filling the right half of the abdomen; the tumour was firm, elastic, and freely moveable. A second tumour similar in character, freely moveable, but of smaller size, was felt in the left lumbar region. By vaginal examinaticn a large firm elastic tumour was felt, filling the right lateral fornix of the vagina, the cervix being small and displaced towards the left side. The tumour appeared to be situated in the right lateral wall of the uterus. As the patient was in poor health she was kept in the hospital for three weeks prior to operation in order that her physical condition might be improved.

On June 21st, at 8 A.M., chloroform was administered. A dose of castor-oil had been given the night before and a rectal enema and vaginal douche had been administered early in the morning. The abdomen had been thoroughly cleaned and asepticised and the pubes shaved on the previous day and the urine had been drawn off just before the operation. An incision was made in the mid-line from a point two inches below the umbilicus to about one inch above the symphysis and the abdominal cavity was opened; some ascitic fluid escaped. The tumours were seen to be dense, solid fibroids attached to the uterine wall by 
short pedicles. The incision had to be enlarged in an upward direction to a point two inches above the umbilicus before the large tumour could be got out of the abdominal cavity. Its surface was covered with apron-like folds of peritoneal membrane through which coursed tortuous vessels of large size, and the omentum was adherent to it in several places. The large vessels were ligatured and cut, the adhesion bands were torn through, and the pedicle, which was about one inch long and as thick as a finger, was ligatured close to the uterus and cut and the tumour was removed. The smaller tumour on the left side was removed in a similar manner. A third tumour, of about the size of a fowl's egg, was found attached by a short pedicle to the posterior wall of the uterus under and behind the large tumour, and this was also ligatured and removed. A fourth large fibroid tumour was now clearly defined, of about the size of a man's fist, in the right half of the pelvis, apparently arising from and in the right lateral wall of the uterus, and situated between the folds of the broad ligament. It was found to be impossible to remove this large sessile tumour without also removing the uterus, and as it was not deemed to be justifiable to increase the patient's risk it was left in situ. Bleeding from the stump of the pedicle of the large tumour gave trouble owing to the ligature having slipped from retraction, but it was eventually controlled by stout silk ligatures. The abdominal cavity was wiped out with sponges but very little blood had escaped into it. The wound was then closed by approximating the edges of the peritoneum with a continuous fine silk suture and the abdominal wall by deep and superficial silk sutures. No drainage-tube was inserted. Tension was aroided by long strips of plaster from loin to loin. A morphia suppository was administered and she was put into bed between blankets with hotwater bottles on each side. The operation was completed and the patient put into bed at 10 A.M.

The patient was only allowed to have small quantities of warm water at intervals until 10 P.M., when milk and barley-water were given every two hours. The urine was drawn off by catheter every four hours. On the 22nd, 23rd, and 24th a raginal douche was given each evening. Before noon each day the temperature remained normal but each afternoon and evening it rose to between $100.4^{\circ}$ and $1008^{\circ} \mathrm{F}$., the pulse remaining at 74 . The patient remained comfortable and cheerful. On the evening of the 24th small repeated doses of sulphate of magnesia were given and the diet was more generous. On the 25 th the morning temperature was $101 \cdot 2^{\circ}$ and the pulse was 76 . The bowels acted, a copious loose stool with much flatus being passed, the patient sleeping well afterwards. The urine was clear. The wound was dressed and was healing well. At 4 P.M. a slight, bloody, offensive discharge came from the vagina; there was no pain. The temperature was still above $101^{\circ}$. On the 26 th the morning temperature was $99 \cdot 6^{\circ}$ and the pulse was 72. The patient passed a good stool without pain. There was a bloody vaginal discharge. The evening temperature was $101.4^{\circ}$ and the pulse was 74 . On the 27 th the morning temperature was $100.4^{\circ}$ and the pulse was 72 . A copious bloody discharge was still present-bright arterial blood; there was no pain. The patient felt well. The evening temperature was $101.6^{\circ}$ and the pulse was 74 . On the 28 th the morning temperature was $100 \cdot 8^{\circ}$ and the pulse was 74. The hæmorrhage from the vagina was less in quantity. An enema was given and a slight amount of flatus was passed. The evening temperature was $101.2^{\circ}$ and the pulse was 70. Ice was applied locally in the vagina. On the 29 th the morning temperature was $99^{\circ}$ and the pulse was 70 . At 4 A.M. a sero-purulent discharge came from the lower end of the wound and soaked the dressings. There was a firm lump felt in the uterine region. The lower stitches were removed to allow free exit for discharge. A probe inserted in the sinus passed upwards and backwards in the mid-line. The hæmorrhage from the vagina was much less. The evening temperature was $99 \cdot 2^{\circ}$ and the pulse was 70 . On the 30 th the morning temperature was $98.2^{\circ}$ and the pulse was 70 . Sweet pus was still discharging from the sinus. The lump in the uterine region was smaller. The temperature was normal all day. The vaginal discharge had almost ceased. On July 1st all the sutures were taken from the wound except three deep middle ones. After this the discharge gradually ceased and the temperature and the pulse remained normal. The patient convalesced rapidly, made an uninter. rapted recovery, and was discharged from the hospital on August 13th.

Remarks by Dr. EarLE.-Case 1 was undoubtedly one of invagination of the sigmoid portion of the colon into the rectum, with prolapse of the invaginated bowel through the anus. The lower two or three inches of the rectum were not prolapsed, as was proved by the insertion of the finger through the anus between the sphincter and the tumour, when the fold between the two layers of rectal mucous membrane could be easily felt round the whole circumference of the tumour. The extreme age ( 89 years) and the weak condition of the patient, the large amount of prolapsed bowel, and the interval that elapsed before the prolapsed bowel was replaced, namely, 22 hours, all tended to increase the gravity of the patient's condition, and the favourable result must be considered noteworthy, as the mortality rate in these cases amongst the aged and infirm is as a rule very high.

In Case 2 the three tumours weighed respectively $6 \mathrm{lb}$. 6oz, $2 \mathrm{lb} .2 \mathrm{oz}$., and $2 \mathrm{oz}$, and were composed of dense fibrons tissue. The tumour in the right lateral wall of the uterns became much smaller in size before the patient left the hospital. The continued rise in the temperature was, I think, due to the suppuration which occurred in the locality of the large silk ligature, for the temperature became normal as soon as efficient drainage of pus occurred. The temperature rose only once above $101^{\circ}$ and the pulse never went beyond 80 . I was ably assisted during the operation by Dr. C. M. Phillips of Philadelphia and Sister Annie Douglass of St. Bartholomew's Hospital, to whom I desire to convey my thanks for their valuable aid.

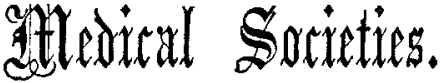

\section{HUNTERIAN SOCIETY.}

\section{Exhibition of Cases.}

A CLINICAL meeting of this society was held on April 11th at the London Institution, the President, Dr. DUNDAS GRANT, being in the chair.

Dr. BUTLER HARRIS showed a case for Diagnosis-an infant with a Sublingual Swelling.-Dr. F. J. SMITH said that he had no doubt that the lump was some form of develop. mental error, most probably a persistent thyroglossal duct. He thought that this was so because of its position in the middle line of the body and from the softish, cystic feeling of the lump. For treatment he advised waiting till the child was a little (a year or more) older and then radical excision.-Dr. RAWES considered it to be a case of venous nævus and he based his diagnosis upon the presence of distended veins in the neighbourhood and upon the fact that the tumour could be so easily compressed between the forefingers.-Mr. J. H. TARGETT suggested that it was a nævoid growth.

Sir HUGH BeEvor showed a case of Hemianopsia occurring in a man 49 years of age. In September, 1897, he had a sudden attack which left him with much loss of sensation on the left side, slight left paralysis, and impaired intelligence. In November, 1897, he was under Dr. Ferrier's care and the record then made was to the effect that his family history had been good and that he had had no ailment but syphilis 17 years before. A few months previonsly le had noticed a sudden loss of sensation on the left side and his eyes were so affected that in his work he ased to spoil material by not cutting it properly; be had noticed little alteration since the attack commenced; the loss of power in his arm and leg had been increasing; he had no loss of consciousness at the time of the attack and had no headache or vomiting. He was a well-built man, looking stupid and listless. Tests of sensation gave the following results: the taste and smell were normal; the hearing was less acute on the left side after removing the cerumen; vision-hemianopsia, left, complete to middle line; analgesia of the left arm and leg; anæsthesia of the face, arm, and leg; and loss of muscular sense in the arm and leg. He walked with a slight stagger and a tendency to fall backwards and to the right. The grasp of his left hand showed loss of power. Ankle-clonus and exaggerated knee-jerk were present. Ophthalmoscopic examination was normal. Cranial tenderness on deep pressure was elicited over the right parietal eminence. In February, 1900, he was attacked 